\title{
From turbulence to financial time series
}

\author{
B. Holdom \\ Department of Physics, University of Toronto \\ Toronto, Ontario, M5S1A7, CANADA \\ holdom@utcc.utoronto.ca
}

\begin{abstract}
We develop a framework especially suited to the autocorrelation properties observed in financial times series, by borrowing from the physical picture of turbulence. The success of our approach as applied to high frequency foreign exchange data is demonstrated by the overlap of the curves in Figure (1), since we are able to provide an analytical derivation of the relative sizes of the quantities depicted. These quantities include departures from Gaussian probability density functions and various two and three-point autocorrelation functions.
\end{abstract}

47.27.Eq, 89.90.+n, keywords: time series, turbulence, autocorrelation

Financial time series and the unexpected scaling behaviors they display have recently attracted the attention [1]-[9] of the theoretical physics community, as large data sets have become available. From a financial times series $x(t)$ and the corresponding set of log price changes $\Delta x \equiv \ln (x(t+\Delta t))-\ln (x(t))$ for time delay $\Delta t$, one constructs the probability density functions (PDFs) $P_{\Delta t}(\Delta x)$. There are three basic observations [泪. 1) $P_{\Delta t}(\Delta x)$ often departs in a leptokurtic manner (sharper peak and close to exponential tails) from the Gaussian shape expected for a random walk. 2) Scale invariance is broken in the sense that the shape of $P_{\Delta t}(\Delta x)$ evolves and gradually becomes more similar to a Gaussian as $\Delta t$ increases. More striking is that this approach to a Gaussian is slower than expected if the individual price changes were independently and identically distributed with finite variance. This anomalous 
scaling indicates that there is some kind of correlation between the price changes at different times (autocorrelation). 3) Although there is negligible autocorrelation observed in the log price changes themselves, there is significant autocorrelation observed in the various functions of the log price changes. It is seen for example in the square of the log price changes, and thus is referred to as volatility clustering.

We make the following definitions, where we assume that the PDFs have mean $\mu=0$ and variance $\sigma^{2}=1$ (otherwise replace $\Delta x$ by $(\Delta x-\mu) / \sigma$, etc.).

$$
\begin{aligned}
& F(f(x), \Delta t)=\frac{\langle f(\Delta x)\rangle}{\langle f(\Delta x)\rangle_{\text {Gauss }}}-1 \\
& G(f(x), \tau)=\frac{\langle f(\Delta x) f(\Delta y)\rangle-\langle f(\Delta x)\rangle^{2}}{\left\langle(f(\Delta x)-\langle f(\Delta x)\rangle)^{2}\right\rangle} \\
& H(f(x), \tau)=\frac{\langle f(\Delta x) f(\Delta y) f(\Delta z)\rangle-2\langle f(\Delta x) f(\Delta z)\rangle\langle f(\Delta x)\rangle+\langle f(\Delta x)\rangle^{3}}{\left\langle(f(\Delta x)-\langle f(\Delta x)\rangle)^{2}\right\rangle^{\frac{3}{2}}}
\end{aligned}
$$

$F$ for various functions $f(x)$ will provide different measures of the departures from a Gaussian PDF, as a function of the time delay $\Delta t . G$ is the autocorrelation function for a lag time $\tau$ where $\Delta x$ is as above and $\Delta y \equiv \ln (x(t-\tau+\Delta t))-\ln (x(t-\tau)) . H$ is a three-point autocorrelation function where $\Delta z \equiv \ln \left(x\left(t-\frac{\tau}{2}+\Delta t\right)\right)-\ln \left(x\left(t-\frac{\tau}{2}\right)\right)$, and it is constructed so that it receives no contributions from autocorrelations on scales smaller than $\tau$. Note that for the autocorrelations, $\Delta t$ is typically constrained to be much less than $\tau$. F, G and $H$ all vanish for a Gaussian random walk.

We consider the data set made freely available in association with the Santa Fe Competition [10]. It consists of 329112 quotes (bid and ask) of the Swiss franc-US dollar exchange rate between May 20, 1985 to April 12, 1991. The time between price quotes is on average a few minutes, but it is very irregular. From this data we extracted a time series with a fixed time step of 10 minutes and a total of 84914 prices. To do this we averaged the bid and ask quotes, used linear interpolation to determine a price at each time, and eliminated the prices changes between days. 
In Fig. (1) we plot as functions of $\Delta t$ or $\tau$ the quantities $F\left(f_{a}(x), \Delta t\right) / \zeta_{a}, G\left(f_{a}(x), \tau\right) / \xi_{a}$ and $H\left(f_{a}(x), \tau\right) / \kappa_{a}$ for $a=1,2, \ldots, 6$. The functions $f_{a}(x)$ and our calculated constants $\left(\zeta_{a}, \xi_{a}, \kappa_{a}\right)$ (which are independent of the normalization of $f_{a}(x)$ ) are specified as follows, where $r \equiv|x|$.

\begin{tabular}{|c|c|c|c|c|c|c|}
\hline$f_{a}(x)$ & $r^{1 / 4}$ & $\sqrt{r}$ & $\frac{1}{1+r}$ & $\frac{1}{3+r}$ & $\frac{1}{1+r^{2}}$ & $\frac{1}{(1+r)^{2}}$ \\
\hline$\zeta_{a}$ & -.0574 & -.0944 & .0772 & .0315 & .0961 & .159 \\
\hline$\xi_{a}$ & .286 & .310 & .299 & .315 & .309 & .270 \\
\hline$\kappa_{a}$ & 1.026 & .617 & 1.045 & 2.129 & .815 & .586 \\
\hline
\end{tabular}

The striking result is that the quantities plotted in Fig. (1) are nearly independent of the function $f_{a}(x)$. This paper will provide an understanding of this result through the derivation of the $\left(\zeta_{a}, \xi_{a}, \kappa_{a}\right)$ constants. From the range of these constants we see that the functions $f_{a}(x)$ are probing the characteristics of the price changes in different ways. Our method also applies to any other functions of choice, and at the end we shall comment on functions which are more sensitive to very large price changes.

We will motivate a one parameter family of PDFs with the following properties. 1) They are derived in closed form. 2) They are leptokurtic with exponential tails. 3) They are closed under convolution. 4) Their derivation is linked to autocorrelation.

The similarity between PDFs in finance and turbulence has recently been emphasized in [2]. For turbulence in a fluid, PDFs are obtained for the velocity differences at two points separated by some distance. The leptokurtic shapes of these PDFs is a reflection of the intermittency of turbulent flow [1]. The origin of this intermittency lies in the coming and going of coherent structures of various sizes, or eddies, in the velocity field. The essential point is that volatility in the velocity differences can be correlated over distance scales typical of the sizes of the eddies. It is the idea of 'volatility structures' of varying time scales, rather than size, which we will carry 
over to financial time series, where price changes replace velocity differences. We will discuss further the analogy between turbulence and financial time series at the end.

To develop a simplified picture we define a volatility structure (VS) of time scale $T$ as follows. Take the total time of the series and split it up into intervals of length $T$. Let the volatility be constant on each interval, but allow it to vary from one time interval to the next. The distribution of these values defines a PDF for volatilities on scale $T$. Our goal is to account for the cumulative effects of VSs occurring on all time scales.

First we consider the effect of a single VS of time scale $T$ on the PDF $P_{\Delta t}(\Delta x)$ for $T \gg \Delta t$. We consider all pairs of prices at times differing by $\Delta t$; over each of these $\Delta t$ intervals the volatility will be constant (ignoring those few intervals which happen to straddle a boundary), but the value of this volatility can be different for widely separated intervals. Thus the PDF will follow by summing over all random walks of duration $\Delta t$ for all values of the constant volatility. This leads to a superposition of Gaussian PDFs,

$$
\mathcal{P}_{\eta}(\sigma, \Delta x)=\int_{0}^{\infty} p_{\eta}(S) P_{\text {Gauss }}(S \sigma, \Delta x) d S
$$

where $P_{\text {Gauss }}(\sigma, \Delta x)=\frac{1}{\sqrt{2 \pi} \sigma} e^{-\frac{(\Delta x)^{2}}{2 \sigma^{2}}}$ and $\sigma^{2}$ is the variance.

$p_{\eta}(S)$ with $S \geq 0$ is the PDF for the volatility. To produce a family of PDFs with the properties listed above we make the following choice.]

$$
p_{\eta}(S)=\frac{2 \eta^{\eta}}{\Gamma(\eta)} S^{2 \eta-1} e^{-\eta S^{2}}
$$

We note that this includes the simple Gaussian, $p_{\frac{1}{2}}(S)=\sqrt{\frac{2}{\pi}} e^{-\frac{S^{2}}{2}}$. The volatility PDF has a mean which approaches unity from below as $\eta$ increases, while its variance

\footnotetext{
${ }^{1}$ An alternative choice for $p_{\eta}(S)$, proposed in both the financial [12] and turbulence [13] literature and advocated more recently [2, [], is the lognormal PDF. The drawback is that the resulting family of PDFs cannot be obtained in closed form, and they are not closed under convolution in the sense we describe.
} 
about this mean approaches

$$
\sigma_{\mathrm{vol}}^{2} \approx \frac{1}{4 \eta}
$$

Thus for large $\eta, p_{\eta}(S)$ becomes sharply peaked around $S=1$ and $P_{\eta}$ tends towards $P_{\text {Gauss }}$.

The integral in (5) can be evaluated to yield $\mathcal{P}_{\eta}(\sigma, \Delta x)=\mathcal{Q}_{1, \eta}(\sigma, r)$ where $r=$ $|\Delta x|, \mathcal{Q}_{i, \eta}(\sigma, r)=\mathcal{Q}_{i, \eta}(1, r / \sigma) / \sigma$ and

$$
\mathcal{Q}_{i, \eta}(1, r)=\frac{1}{\pi^{\frac{i}{2}} \Gamma(\eta)} 2^{1-\frac{i}{4}-\frac{\eta}{2}} \eta^{\frac{i}{4}+\frac{\eta}{2}} r^{\eta-\frac{i}{2}} K_{\eta-\frac{i}{2}}(\sqrt{2 \eta} r) .
$$

$K_{\nu}(x)$ is the modified Bessel function of the second kind and $i \neq 1$ will be of interest below. These PDFs have a distinctive leptokurtic shape which becomes more prominent for decreasing $\eta$. For any positive integer $n, \mathcal{P}_{n}(1, \Delta x)$ is simply a polynomial in $|\Delta x|$ of degree $n-1$ times the exponential $e^{-\sqrt{2 n}|\Delta x|}$. The appearance of these exponential tails can be traced to the Gaussian factor in (6). The simplest member of this family of PDFs is purely exponential, $\mathcal{P}_{1}(1, \Delta x)=\frac{1}{\sqrt{2}} e^{-\sqrt{2}|\Delta x|}$.

We now consider the PDF $P_{\Delta t}(\Delta x)$ which results from a single VS with $T<\Delta t$. For example with $\Delta t=n T$ we must consider the convolution

$$
\int d x_{1} \ldots d x_{n-1} \mathcal{P}_{\eta}\left(\sigma, x_{n}-x_{n-1}\right) \mathcal{P}_{\eta}\left(\sigma, x_{n-1}-x_{n-2}\right) \ldots \mathcal{P}_{\eta}\left(\sigma, x_{1}-x_{0}\right)
$$

where the $x_{i}$ are the $\log$ prices at regularly spaced intervals $t_{i}-t_{i-1}=T$. This convolution accounts for the variable volatility on each interval. We now make use of the fact that our family of PDFs is closed under convolution. In particular the results of [14] are sufficient to prove the following relation for half-integer $\eta_{1}$ and $\eta_{2}$.

$$
\int d y \mathcal{P}_{\eta_{1}}\left(\sqrt{\eta_{1}} \sigma, x-y\right) \mathcal{P}_{\eta_{2}}\left(\sqrt{\eta_{2}} \sigma, y-z\right)=\mathcal{P}_{\eta_{1}+\eta_{2}}\left(\sqrt{\eta_{1}+\eta_{2}} \sigma, x-z\right)
$$

Thus the $\mathrm{PDF} P_{\Delta t}(\Delta x)$ determined by $(9)$ is $\mathcal{P}_{n \eta}\left(\sqrt{n} \sigma, x_{n}-x_{0}\right)$. 
There are three points worth stressing for this case of a VS with $T<\Delta t$. 1) The variance of $P_{\Delta t}(\Delta x)$ increases exactly linearly with $\Delta t$ for fixed $T$. (This is trivially true for the previous case, $T \gg \Delta t$.) This linear growth of variance is a well documented feature of financial time series [4], which can be related to the vanishing of $G(f(x), \tau)$ for $f(x)=x$ [6]. 2) From the relation (17) between $\sigma_{\text {vol }}^{2}$ and $\eta$ we see that the effective variance in volatilities $\sigma_{\text {vol }}^{2}$ on time scale $\Delta t$ is decreasing as $1 / \Delta t$. In other words as the number $n$ of intervals with independently fluctuating volatilities increases the effective PDF tends towards a Gaussian, in a manner as expected from the central limit theorem [15]. 3) We now see that the whole family of PDFs in (运) are nothing but convolutions of the simple case when the volatility $\operatorname{PDF} p_{\eta}(S)$ is a Gaussian $(\eta=1 / 2)$.

We next consider the autocorrelation between price changes $\Delta x$ and $\Delta y$, each occurring over a time step $\Delta t$, but separated by a lag $\tau>\Delta t$. For a VS with $T \gg \tau$ we must sum over all pairs of random walks of duration $\Delta t$ for all values of a common volatility. The joint PDF is then

$$
\mathcal{J}_{\eta}(\sigma, \Delta x, \Delta y)=\int_{0}^{\infty} p_{\eta}(S) P_{\text {Gauss }}(S \sigma, \Delta x) P_{\text {Gauss }}(S \sigma, \Delta y) d S
$$

More generally an $i$-point joint PDF can be defined in the corresponding way, and we obtain

$$
\mathcal{J}_{\eta}\left(\sigma, \Delta x_{1}, \Delta x_{2}, \ldots, \Delta x_{i}\right)=\mathcal{Q}_{i, \eta}\left(\sigma, \sqrt{\left(\Delta x_{1}\right)^{2}+\left(\Delta x_{2}\right)^{2}+\ldots+\left(\Delta x_{i}\right)^{2}}\right)
$$

where $\mathcal{Q}_{i, \eta}(1, r)$ is defined in (8).

From this joint PDF we may extract for example the PDF for the following combination of the $m$ adjacent $\Delta x$ 's within time $m \Delta t: v_{m} \equiv \sqrt{\sum_{\text {adjacent }}(\Delta x)^{2}}$. 2 Thus for

\footnotetext{
${ }^{2} \mathrm{~A}$ very similar quantity was recently studied in 9 .
} 
VSs with $T \gg m \Delta t$ we obtain the $\operatorname{PDF} \mathcal{R}_{m, \eta}\left(v_{m}\right)=2 \pi^{\frac{m}{2}} v_{m}{ }^{m-1} \mathcal{Q}_{m, \eta}\left(\sigma, v_{m}\right) / \Gamma\left(\frac{m}{2}\right)$. This cannot be directly compared with data because it does not account for VSs with $T \lesssim m \Delta t$

We return to the quantities $F, G$, and $H$ in (1-3), for which we can account for VSs occurring on all time scales $T$. For $G$ and $H$ only VSs with $T$ larger than $\tau$ contribute, and the cumulative effect of such VSs can be represented by an effective $\sigma_{\text {vol }}^{2}(\tau)$. From the inverse relation (17) between $\sigma_{\text {vol }}^{2}$ and $\eta$ this corresponds to an effective $\eta$ which will specify $\mathcal{J}_{\eta}(\sigma, \Delta x, \Delta y, \ldots)$, which in turn enters the determination of $G$ and $H$. $G$ and $H$ vanish only in the large $\eta$ limit.

For the PDF $P_{\Delta t}(\Delta x)$, VSs of time scales both larger and smaller than $\Delta t$ contribute. We have seen how each VS with $T<\Delta t$ makes a contribution to an effective $\sigma_{\text {vol }}^{2}(\Delta t)$ suppressed by $T / \Delta t$. These contributions can be added to the contribution of VSs larger than $\Delta t$ to yield a total effective $\sigma_{\mathrm{vol}}^{2}(\Delta t)$. The corresponding effective $\eta$ then specifies a $\operatorname{PDF} \mathcal{P}_{\eta}(\sigma, \Delta x)$ which can be used to determine $F$. Unlike the case of a single VS with $T<\Delta t$, the effective $\sigma_{\text {vol }}^{2}(\Delta t)$ may now fall slower than $1 / \Delta t$. This corresponds to the slow approach to a Gaussian, which is the anomalous scaling observed in the data. Thus in our picture it is the presence of VSs on all time scales which underlies the link [4, 6] between this anomalous scaling and the nonvanishing autocorrelations.

To compare with data we first use the 1, 2, and 3-point PDFs, $\mathcal{P}_{\eta}$ and $\mathcal{J}_{\eta}$, to determine $F, G$, and $H$ as functions of $\eta$.

$$
F\left(f_{a}(x), \eta\right)=\frac{\zeta_{a}(\eta)}{\eta}, \quad G\left(f_{a}(x), \eta\right)=\frac{\xi_{a}(\eta)}{\eta}, \quad H\left(f_{a}(x), \eta\right)=\frac{\kappa_{a}(\eta)}{\eta}
$$

We find that $\zeta_{a}(\eta), \xi_{a}(\eta)$ and $\kappa_{a}(\eta)$ are rather slowly varying functions which tend to constants for large $\eta$. In principle we could extract $F\left(f_{a}(x), \Delta t\right), G\left(f_{a}(x), \tau\right)$, and 
$H\left(f_{a}(x), \tau\right)$ from the data and by comparing with (13), determine the appropriate $\eta$ for each $\Delta t$ and $\tau$ respectively. The prediction would be that the values of $\eta$ and the values of $F / \zeta, G / \xi$ and $H / \kappa$ are independent of $f_{a}(x)$. In fact for the range of $\eta$ we are considering we can, to good approximation, replace the functions $\zeta_{a}(\eta), \xi_{a}(\eta)$ and $\kappa_{a}(\eta)$ by constants. The values listed in (4) correspond to $\left(\zeta_{a}(3 / 2), \xi_{a}(3 / 2), \kappa_{a}(3 / 2)\right)$, which in turn yield the results in Fig. (1). In addition to the overlap of the curves we also note the close similarity of Figs. (1B) and (1C). This strongly supports our common description of all $i$-point autocorrelation functions in terms of the function $\mathcal{Q}_{i, \eta}(\sigma, r)$ in $(\mathbb{8})$.

In general our model provides a good description of the PDFs and the autocorrelations, with the exception being quantities sensitive to abnormally large price changes. For example when $F / \zeta$ with $f(x)=x^{4}$ is extracted from the data we obtain the top curve in Fig. (1A). $\left(F\left(x^{4}, \eta\right)\right.$ is directly related to the kurtosis of our PDFs, and in this case we have simply $\zeta(\eta)=1$.) This indicates that the extreme tails of the true PDFs fall off somewhat slower than expected, especially for shorter time delays. More striking are the autocorrelations involving large price changes; for example $G / \xi$ and $H / \kappa$ with $f(x)=x^{2}$ gives the bottom curves in Figs. (1B) and (1C) respectively. Thus large price changes show much less autocorrelation than expected. This suggests that large price changes more strongly reflect the reaction of the markets to external events.

We conclude by commenting on the analogy with turbulence in a fluid [2, 3, 4, 5, 8]. The PDFs for turbulence evolve as a function of the distance $r$ separating the two points at which the velocities are measured. We have presented a model for this evolution based on a formulation related to the present work [14]. A basic observation is that the evolution becomes more transparent when expressed in terms 
of $\tau \propto r^{2 / 3}$. (In fact $\tau$ is a physical time scale in turbulence, the so-called turnover time [11].) In particular the variance of the PDF is proportional to $\tau$, while its shape evolves from the leptokurtic towards the Gaussian as $\tau$ increases, making the analogy between financial time series and turbulence very close. The difference for turbulence in a fluid is that the data is quite consistent [14] with a speed of approach to the Gaussian as described by the central limit theorem, $\sigma_{\mathrm{vol}}^{2} \propto 1 / \tau$. Thus contrary to financial time series, volatility structures (eddies in this case) on large $\tau$ scales are not sufficiently strong to cause a significant slowing down in the approach to the Gaussian. The turbulence in the financial markets is stronger in this sense than the turbulence observed in a fluid.

\section{Acknowledgement}

This research was supported in part by the Natural Sciences and Engineering Research Council of Canada.

Figure (1): A, B, and C each display six curves with significant overlap. $F\left(f_{a}(x), \Delta t\right) / \zeta_{a}$, $G\left(f_{a}(x), \Delta t\right) / \xi_{a}$ and $H\left(f_{a}(x), \Delta t\right) / \kappa_{a}$ are defined in (11-3) with the six functions $f_{a}(x)$ and constants $\left(\zeta_{a}, \xi_{a}, \kappa_{a}\right)$ specified in (4). The additional dashed line in A corresponds to $f(x)=x^{4}$, while the dashed line in each of $\mathrm{B}$ and $\mathrm{C}$ corresponds to $f(x)=x^{2}$. To produce $\mathrm{B}$ and $\mathrm{C}$ we have removed from the data the well known correlation between volatility and time of day. This changes the shape of the curves but not their overlap. 


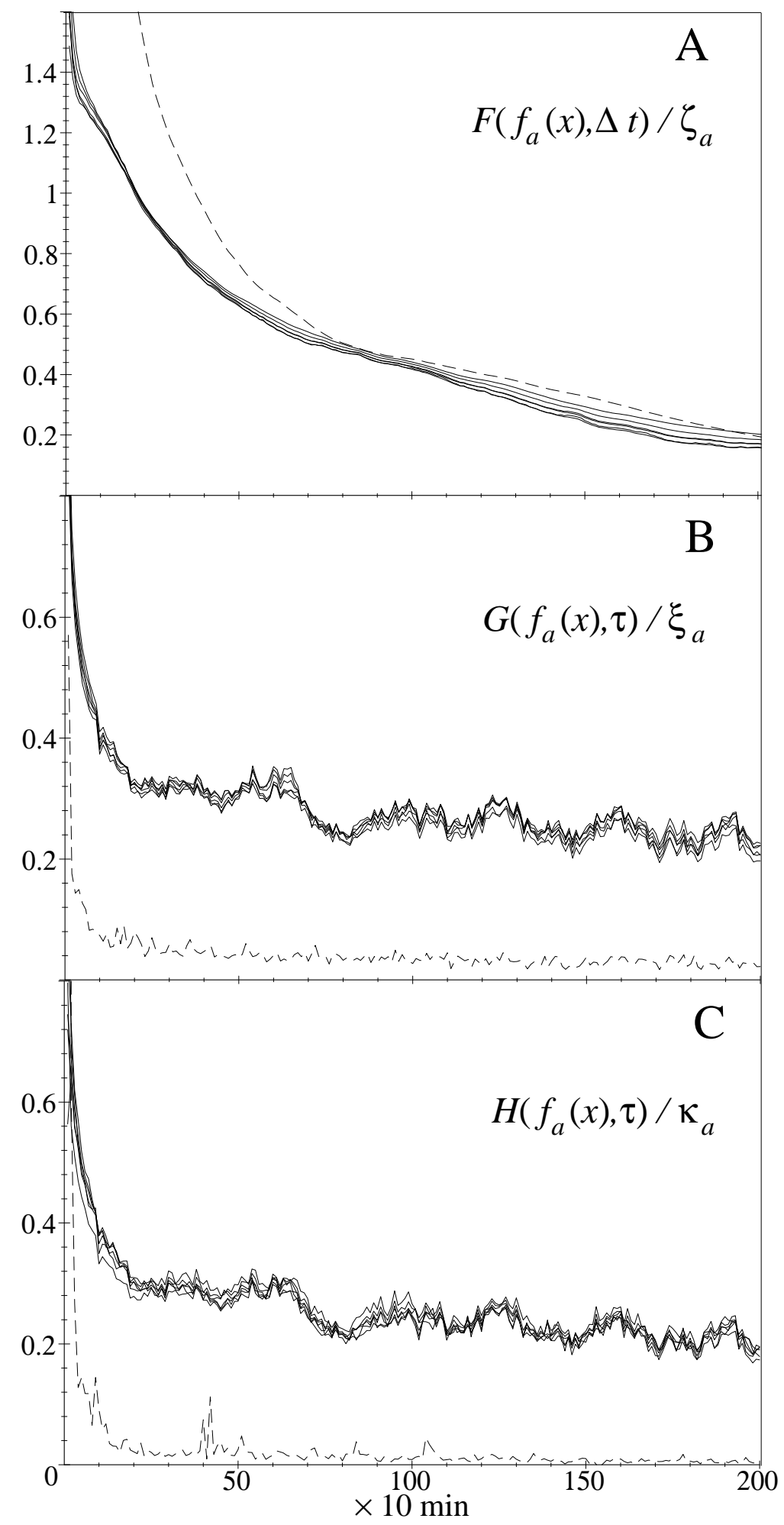




\section{References}

[1] R. Mantegna and H.E. Stanley, "Scaling behavior in the dynamics of an economic index", Nature 376 (1995) 46.

[2] S. Ghashghaie, W. Breymann, J. Peinke, P. Talkner, and Y. Dodge, "Turbulent cascades in foreign exchange markets", Nature 381 (1996) 767.

[3] R. Mantegna and H.E. Stanley, "Turbulence and financial markets", Nature 383 (1996) 587; "Stock market dynamics and turbulence: parallel analysis of fluctuation phenomena", Physica A239 (1997) 255.

[4] R. Cont, M. Potters, J.-P. Bouchaud, "Scaling in stock market data: stable laws and beyond", in Scale Invariance and Beyond, proceedings of the CNRS Workshop on Scale Invariance, Les Houches, March 1997, cond-mat/9705087.

[5] A. Arneodo, J.-P. Bouchaud, R. Cont, J.-F. Muzy, M. Potters, and D. Sornette, "Comment on turbulent cascades in foreign exchange markets, preprint", condmat/9607120.

[6] R. Cont, "Scaling and correlation in financial time series: a study of S\&P futures prices", preprint, cond-mat/9705075.

[7] Y. Liu, P. Cizeau, M. Meyer, C.-K. Peng, and H.E. Stanley, "Correlations in economic time series", preprint, cond-mat/9706021.

[8] A. Arneodo, J.-F. Muzy and D. Sornette, "Causal cascade in the stock market from the infrared to the ultraviolet", cond-mat/9708012.

[9] P. Cizeau, Y. Liu, M. Meyer, C.-K. Peng, and H.E. Stanley, "Volatility distribution in the S\&P500 Stock Index", cond-mat/9708143. 
[10] Time Series Prediction: Forecasting the Future and Understanding the Past, A. S. Weigend and N. A. Gershenfeld, eds., Addison-Wesley, 1993.

[11] U. Frisch, Turbulence: The Legacy of A. N. Kolmogorov, Cambridge University Press, New York, 1995.

[12] P. Clark, "A Subordinated stochastic process model with finite variance for speculative prices", Econometrica 41(1) (1973) 135.

[13] B. Castaing, Y. Gagne, and E. Hopfinger, "Velocity probability density functions of high Reynolds number turbulence", Physica D46 (1990) 177; A. N. Kolmorgorov, J. Fluid Mech. 13 (1962) 82; A. M. Obukhov, J. Fluid Mech. 13 (1962) 77.

[14] B. Holdom, "Intermittency and the slow approach to Kolmogorov scaling", Phys. Rev. E55 (1997) 7000.

[15] W. Feller, An Introduction to Probability Theory and Its Applications, Wiley, New York, 1971. 